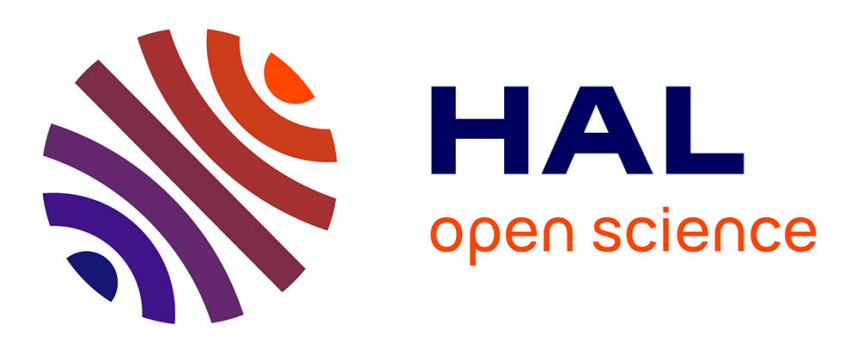

\title{
How to change or remove the anisotropy of spin glasses, with some other reflections on the anisotropy problem
}

\author{
A. Fert, S. Senoussi, D. N. Arvanitis
}

\section{To cite this version:}

A. Fert, S. Senoussi, D. N. Arvanitis. How to change or remove the anisotropy of spin glasses, with some other reflections on the anisotropy problem. Journal de Physique Lettres, 1983, 44 (9), pp.345350. 10.1051/jphyslet:01983004409034500 . jpa-00232202

\section{HAL Id: jpa-00232202 https://hal.science/jpa-00232202}

Submitted on 1 Jan 1983

HAL is a multi-disciplinary open access archive for the deposit and dissemination of scientific research documents, whether they are published or not. The documents may come from teaching and research institutions in France or abroad, or from public or private research centers.
L'archive ouverte pluridisciplinaire HAL, est destinée au dépôt et à la diffusion de documents scientifiques de niveau recherche, publiés ou non, émanant des établissements d'enseignement et de recherche français ou étrangers, des laboratoires publics ou privés. 
Classification

Physics Abstracts

$75.30 \mathrm{H}-75.30 \mathrm{E}-75.30 \mathrm{G}$

\title{
How to change or remove the anisotropy of spin glasses, with some other reflections on the anisotropy problem
}

\author{
A. Fert, S. Senoussi and D. Arvanitis \\ Laboratoire de Physique des Solides (*), Université Paris-Sud, 91405 Orsay, France
}

(Reçu le 24 janvier 1983, accepté le 16 mars 1983)

\begin{abstract}
Résumé. - Nous développons quelques idées sur le gel de l'énergie d'anisotropie macroscopique des verres de spins et nous introduisons les notions d'anisotropie thermo-rémanente et d'anisotropie rémanente isotherme. Nous présentons des résultats de cycle d'hystérésis sur des alliages CuMn qui confirment ces idées. En particulier, nous décrivons la recette qui nous permet de réduire à volonté et même de supprimer l'anisotropie.
\end{abstract}

\begin{abstract}
We develop some ideas on the freezing of the macroscopic anisotropy energy of spin glasses and introduce the concepts of thermo-remanent anisotropy (TRA) and isothermal remanent anisotropy (IRA). We present results of hysteresis cycle experiments on CuMn alloys which confirm these ideas. In particular we describe a method which allows us to reduce the anisotropy at will and even to remove it.
\end{abstract}

The macroscopic anisotropy of spin glasses such as CuMn, AgMn or NiMn has been shown to be a very interesting property for its character of memory effect and its outstanding tridirectional symmetry [1-5]. The anisotropy is generally thought to arise from the freezing of correlations between the spins and local anisotropy fields due to the presence of Dzyaloshinsky-Moriya interactions. In this letter we develop some ideas on the freezing of the anisotropy (in particular we introduce the concepts of thermo-remanent anisotropy and isothermal remanent anisotropy) and present hysteresis cycles experiments in support of our ideas. We acknowledge that some of our ideas arise from very enlightening discussions with Christofer Henley.

The starting point of the interpretation of the macroscopic anisotropy of spin glasses by Levy et al. [1-6], Henley et al. [2], or Fert and Hippert [4] is the existence of microscopic DzyaloshinskyMoriya (DM) interactions.

$$
E_{\mathrm{DM}}=\sum_{i j} \mathbf{D}_{i j} \cdot\left(\mathbf{S}_{i} \times \mathbf{S}_{j}\right) .
$$

The vectors $\mathbf{D}_{i j}$ are tied to the lattice. They are randomly oriented. A macroscopic anisotropy energy exists because correlations between the vectors $\mathbf{S}$ and $\mathbf{D}$ are frozen during the cooling below $T_{g}$ (more precisely : among many possible ground states which would be equivalent in the

(*) Laboratoire associé au CNRS. 
absence of DM interactions, the spin glass preferably chooses those with $E_{\mathrm{DM}}<0$ ). This macroscopic anisotropy can be called thermo-remanent anisotropy (TRA) by analogy with the thermoremanent magnetization (TRM) which is due to the freezing of correlations between the spins and the direction of the magnetic field applied during the cooling. A difference between TRA and TRM is that a spin glass is always cooled in the presence of the same local anisotropy fields whereas the field applied during the cooling can be choosen at will. This explains that conventional experiments find a unique anisotropy constant for a given spin glass at a given temperature (after zero field cooling or field cooling, and whatever the cooling field) [1, 5], while the TRM depends on the cooling field. However there is a way to change the local anisotropy fields seen by the spins during their cooling : this is by rotating the lattice (and therefore the vectors $\mathbf{D}_{i j}$ ) with respect to the spin system or, equivalently, the spin system with respect to the lattice. We now describe experiments based on this idea which change the macroscopic anisotropy and confirm our general picture.

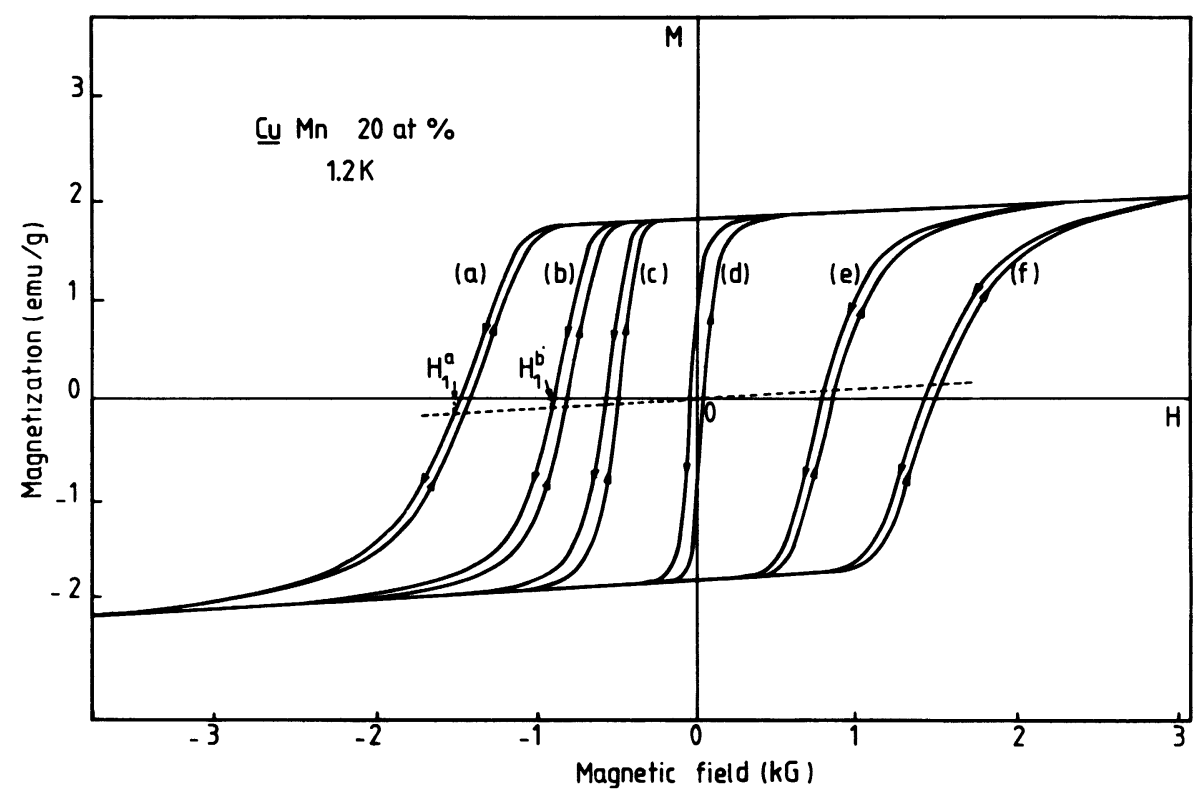

Fig. 1. - Series of hysteresis cycles of CuMn 20 at \% recorded at $1.2 \mathrm{~K}$ under the following conditions. Curve (a) : after cooling the sample down to $1.2 \mathrm{~K}$ in $9.1 \mathrm{kG}$, the cycle is recorded by simply sweeping the field between $\pm 9.1 \mathrm{kG}$. Curves (b), (c), (d), (e), (f) : stopping the cycle (a) at $-9.1 \mathrm{kG}$, we heated the sample up to $T($ (b) $: T=11 \mathrm{~K}$, (c) $: T=24 \mathrm{~K}$, (d) $: T=31 \mathrm{~K}$, (e) $: T=60 \mathrm{~K}$, (f) $: T=88 \mathrm{~K}$ ) and cooled it back to $1.2 \mathrm{~K}$. Then the cycle was recorded by sweeping the field between $\pm 9.1 \mathrm{kG}$. The reversal fields $H_{1}^{\mathrm{a}}, H_{1}^{\mathrm{b}}$, etc..., are defined as the fields where the curves a, b, etc..., intercept the dashed line $M=\chi H$ where $\chi$ is the reversible susceptibility.

By using a Foner magnetometer [7] we recorded series of hysteresis cycles of a CuMn 20 at \% alloy at $1.2 \mathrm{~K}$ under the following conditions. After cooling the sample down to $1.2 \overline{\mathrm{K}}$ in $+9.1 \mathrm{kG}$, we first recorded its hysteresis cycle simply by sweeping the field down to $-9.1 \mathrm{kG}$ and back to $+9.1 \mathrm{kG}$. As previously observed in concentrated CuMn alloys [8], we obtained a displaced cycle with a very narrow width (curve (a) in Fig. 1). This typical shape corresponds to a directional anisotropy energy of the form $K(1-\cos \theta)$ and the anisotropy constant is related to the reversal field $H_{1}$ by $K=-H_{1} / \mu_{\mathrm{r}}\left(\mu_{\mathrm{r}}=\right.$ remanent magnetization) [8,9]. In subsequent experiments we stopped the preceding field sweep at $-9.1 \mathrm{kG}$, heated the sample up to $T\left(T<T_{\mathrm{g}}=98 \mathrm{~K}\right)$, cooled it back to $1.2 \mathrm{~K}$ and then recorded the cycle as above by sweeping the field up and down 
between + and $-9.1 \mathrm{kG}$. The curves (b), (c), (d), (e) and (f) of figure 1 show the cycles (narrow, displaced and qualitatively similar to the initial cycle of curve (a)) recorded for different values of $T$. The remanent magnetization is always the same as that in the initial cycle (curve (a)) but, in contrast, the anisotropy constant decreases when $T$ increases. It goes to zero for about $T \sim 30 \mathrm{~K}$ and is negative for higher values of $T$. For $T \gtrsim 88 \mathrm{~K}$ the anisotropy constant saturates at the value $-K(1.2)(K(1.2)=$ anisotropy constant of the cycle of curve (a)). The explanation of these results is straightforward :

i) the remanent magnetization is not changed during the heating-cooling because, during this stage, the applied field is oriented in the direction of the remanence (both are negative) and has the value $(9.1 \mathrm{kG})$ of the initial cooling field.

ii) In contrast, during the heating-cooling stage at $-9.1 \mathrm{kG}$, the spins see a new lattice of vectors $\mathbf{D}_{i j}$ (reversed with respect to the spin system [10]). During the heating to $T$, a part of the correlations between the spins and the old vectors $\mathbf{D}_{i j}$ is released (the system jumps over energy barriers by thermal excitations) which gives the well known decrease of anisotropy with temperature. During the cooling back to $1.2 \mathrm{~K}$, correlations with the new vectors $\mathbf{D}_{i j}$ are frozen. In short, a fraction of the positive anisotropy constant $K(1.2)$ is destroyed during the heating, while a negative contribution to $K$ is created during the cooling. This explains that $K$ decreases as $T$ increases and becomes negative at higher values of $T$.

Before presenting a quantitative analysis of the preceding results, we have to discuss more precisely the effects of temperature on the anisotropy. The simplest situation is that at low temperature (i.e. $T \ll T_{\mathrm{g}}$, for example $T \cong 1.2 \mathrm{~K}$ for CuMn 20 at $\%$ ) : the width of the cycle is practically zero (Fig. 1 and $2 a$ ) which corresponds to an anisotropy energy of the form $K(1-\cos \theta)$. At higher temperature the hysteresis loop has a non-zero width (Fig. $2 b$ ) and it has been shown that this broadening goes with time dependent and irreversible rearrangements of the spins during the reversal of the spin system and is not due to an admixture of a true axial anisotropy into the directional anisotropy [11]. In other words, it can be said that the change of the reversal field from $\mathrm{H}_{1}$ to $\mathrm{H}_{2}$ (see Fig. $2 b$ ) is due to an effect of isothermal remanent anisotropy (IRA) adding to the always existing thermo-remanent anisotropy (TRA). As the isothermal remanent magnetization (IRM) is induced by applying a magnetic field isothermally, the IRA is induced by rotating the lattice with respect to the spins or the spins with respect to the lattice at a fixed temperature. It is well known [12] that, at least for small fields, the IRM is negligible with respect to the TRM at $T \ll T_{\mathrm{g}}$ and becomes relatively significant at higher temperature. It appears that, in the same way, the IRA is negligible with respect to the TRA at $T \ll T_{\mathrm{g}}$ (narrow displaced cycle, Fig. $2 a$ ) and becomes significant at higher temperatures (broadened cycle becoming more and more symmetric, Fig. 2b) [13]. At high enough temperature the IRA is saturated and the cycle is symmetric (Fig. 2c). Quantitatively this picture means that, around $\theta=0$, the anisotropy is written :

$$
E_{\mathrm{A}}(\theta)=K^{\mathrm{TRA}}(1-\cos \theta)
$$

with $K^{\mathrm{TRA}}>0$ and, roughly, $H_{1} \cong-K^{\mathrm{TRA}} / \mu_{\mathrm{r}}$, whereas, around $\theta=\pi$,

$$
E_{\mathrm{A}}(\theta)=\left(K^{\mathrm{TRA}}+K^{\mathrm{IRA}}\right)(1-\cos \theta),
$$

with $K^{\mathrm{IRA}}<0$ and, roughly, $H_{2} \cong-\left(K^{\mathrm{TRA}}+K^{\mathrm{IRA}}\right) / \mu_{\mathrm{r}}$.

Taking into account the existence of the IRA component when $T$ increases makes the quantitative analysis of the results of figure 1 a little more complex than naively expected but leads to a better agreement. After the heating at $T$ in the reversed state the anisotropy constant is :

$$
K^{\operatorname{TRA}}(T)+K^{\operatorname{IRA}}(T)
$$




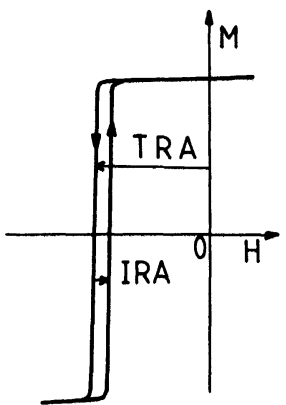

(a): $T \ll T g$

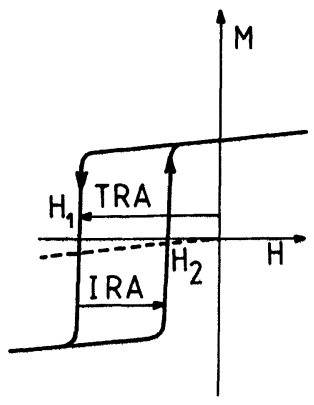

(b)

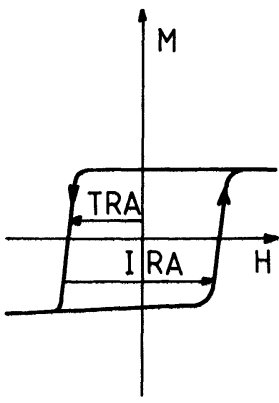

(c)

Fig. 2. - Typical hysteresis curve of CuMn (at $T<T_{\mathrm{g}}$ ) showing contributions from thermo-remanent anisotropy (TRA) and isothermal remanent anisotropy (IRA). (a) : with a very small IRA at $T \ll T_{\mathrm{g}}$; (b) : with a significant IRA at higher temperature; (c) : with a saturated IRA at still higher temperature. The mechanisms of TRA and IRA are explained in the text (minor remark : the above scheme is proposed for cycles after field cooling or zero field cooling. Field cooling creates at the same time a TRA tending to maintain the orientation of the spin triad [3] and a TRM $\mu_{\mathrm{r}}$ along an axis of the spin triad. Zero field cooling creates a TRA tending to maintain the orientation of the spin triad; then applying a field pins a IRM along an axis of the triad. Note that, in the later case, symmetrical sweeps of the field after zero field cooling, can lead to an asymmetrical cycle, which is a frequently observed apparent paradoxe. However the narrow displaced cycle (a) is rarely observed after.zero field cooling because, at $T \ll T_{g}$, usual experimental fields are to small to induce a significant anisotropy).

The subsequent cooling at $1.2 \mathrm{~K}$ in the presence of a reversed DM lattice creates a TRA contribution equal to

$$
-\left(K^{\mathrm{TRA}}(1.2)-K^{\mathrm{TRA}}(T)\right)
$$

which gives for the anisotropy constant after the heating-cooling stage

$$
K^{\prime}(1.2 \leftrightarrow T)=2 K^{\mathrm{TRA}}(T)-K^{\mathrm{TRA}}(1.2)+K^{\mathrm{IRA}}(T) .
$$

This is the anisotropy constant in the reversed state $(\theta \cong \pi)$. If we do not neglect the very small $K^{\text {IRA }}(1.2)$ (proportional to the width of the cycle at $1.2 \mathrm{~K}$ ) the anisotropy in the non-reversed state $(\theta \cong 0)$ is

$$
K(1.2 \leftrightarrow T)=2 K^{\mathrm{TRA}}(T)-K^{\mathrm{TRA}}(1.2)+K^{\mathrm{IRA}}(T)-K^{\mathrm{IRA}}(1.2) .
$$

Although $K^{\operatorname{IRA}}(T)$ and $K^{\text {IRA }}(1.2)$ are not very large, they are needed for a precise analysis of the results. We determined $K^{\text {TRA }}(T)$ and $K^{\text {IRA }}(T)$ by recording hysteresis cycles [14] of our sample after direct cooling from $T_{\mathrm{g}}$ to $T$ and we calculated the values of $K(1.2 \leftrightarrow T)$ and $H_{1}(1.2 \leftrightarrow T)=$ $K(1.2 \leftrightarrow T) / \mu_{\mathrm{r}}$ predicted by equation (7). The agreement with the experimental values of $H_{1}(1.2 \leftrightarrow T)$ is good (Fig. 3). This confirms the overall picture. However we point out that hysteresis cycle experiments are less appropriate for a precise quantitative interpretation than torque $[4,10]$ or transverse susceptibility $[5,10]$ experiments probing the variation of the anisotropy energy as a function of the rotation angle $\theta$. Therefore we expect more quantitative data from torque experiments now in progress at Orsay. The interest of the hysteresis experiments presented in this paper is rather in the clearness of the qualitative results of figure 1.

In conclusion, we have presented experiments confirming that the remanent magnetization and the anisotropy of spin glasses are two similar but independent [15] properties associated with different types of correlations : the remanent magnetization represents correlations between 


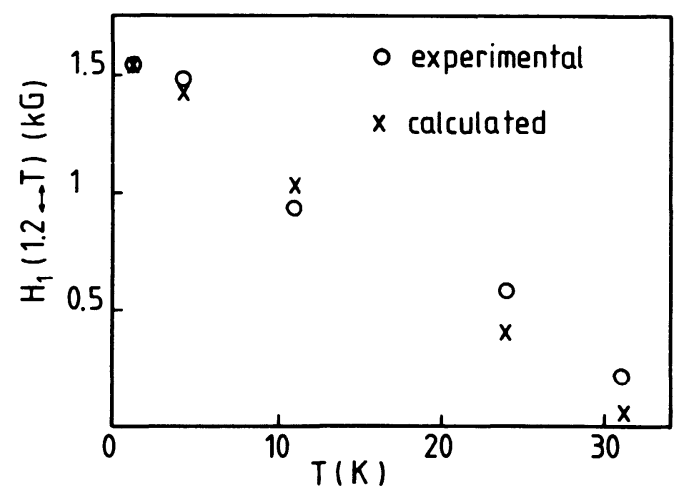

Fig. 3. $-H_{1}(1.2 \leftrightarrow T)$ versus $T$ for CuMn 20 at \% at $1.2 \mathrm{~K}$. The experimental values of $H_{1}(1.2 \leftrightarrow T)$ are the reversal fields of the cycles of figure 1 . Their calculated values are obtained from equation (7), as described in the text. Above about $30 \mathrm{~K}$ the hysteresis cycles are not sharp (abrupt) enough to reliably determine $K^{\text {TRA }}(T)$ and $K^{\text {IRA }}(T)$. Consequently we compare the experimental and calculated values of $K(1.2 \leftrightarrow T)$ only in the range $T \leqslant 31 \mathrm{~K}$.

the spins and the direction of the applied field and the anisotropy represents correlations between the spins and the directions of local anisotropy fields. We have introduced the concepts of TRA and IRA by analogy with the TRM and IRM. Our experiments are also interesting in other aspects. They show that the anisotropy of a spin glass such as CuMn can be erased [16] by appropriate thermal treatment. The possibility of removing the anisotropy may be of definite interest in several situations. For example, in MNR, the enhancement of the r.f. field is inversely proportional to the anisotropy constant [17], so that lowering the anisotropy should make it easier to perform NMR studies in spin glasses. Also, in ESR, it may be of interest to change the anisotropy of a given sample at a given temperature. For example, Monod and Cowen [18] have recently used our method to study the influence of the magnitude of $K$ on the ESR linewidth of CuMn.

\section{Acknowledgments.}

We thank Christofer Henley for very fruitful discussions and Philippe Monod for providing us with one of the samples.

\section{References}

[1] Levy, P. M., Morgan-Pond, C. and Fert, A., J. Appl. Phys. 53 (1982) 2168.

[2] Henley, C. L., Sompolinsky, H. and Halperin, B. I., Phys. Rev. B 25 (1982) 5849.

[3] Saslow, W. M., Phys. Rev. Lett. 48 (1982) 505.

[4] Fert, A. and Hippert, F., Phys. Rev. Lett. 49 (1982) 1508.

[5] Alloul, H. and Hippert, F., ICM 82 - Kyoto, to be published in J. Magn. Magn. Mat.

[6] Fert, A, and Levy, P. M., Phys. Rev. Lett. 44 (1980) 1538.

[7] Details on the experimental techniques will be given elsewhere.

[8] Kouvel, J. S., J. Phys. Chem. Sol. 21 (1961) 57.

[9] Monod, P., Prejean, J. J. and Tissier, B., J. Appl. Phys. 50 (1979) 7324.

[10] Torque experiments on CuMn 20 at \% (Ref. 4), clearly show that, when the remanent magnetization is reversed by inverting the field (from $+z$ to $-z$ ), the spin system breaks into blocks which rotate by $\pi$ around different axes randomly distributed in the plane $x y$. Therefore, with respect to the spins of a given block, the reversal of the lattice of vectors $\mathbf{D}$ is a rotation by $\pi$. 
[11] Hippert, F., Alloul, H. and Fert, A., J. Appl. Phys. 53 (1982) 7702.

[12] Bouchiat, H. and Monod, P., J. Magn. Magn. Mat. 30 (1982) 175.

[13] Of course, this picture of the hysteresis is proposed only for systems with directional anisotropy such as CuMn, AgMn, NiMn. It certainly exists systems in which the width of the cycle arises from a true axial $\left(\sim K_{2} \overline{\sin }^{2} \theta\right)$ anisotropy. And it also exists spin glasses (dilute AuFe alloys, for example) with a very high anisotropy preventing the spin system to rotate, so that the hysteresis is due to a progressive change of the magnetization without rotation and has nothing to see with anisotropy.

[14] The anisotropy constants derived from reversal fields by equations $(2,3)$ are somewhat smaller than those determined from torque measurements but have the same variation with temperature.

[15] The remanent magnetization and anisotropy are independent only if the remanent magnetization does not exceed a small fraction of the saturated magnetization. Obviously, a significant polarization of the spins reduces the possibilities of correlations with local anisotropy fields, then remanence and anisotropy are no longer independent.

[16] Our conclusion that "the anisotropy can be erased " must be qualified. As described in the footnote [10], when the field is reversed, the spin system breaks into several blocks which rotate by $\pi$ around different axes. The results of our hysteresis measurements show that the anisotropy associated with rotations around these axes is erased. But torque [19] and EPR [18] measurements show that the anisotropy for rotation around other axes is changed but not removed. However the anisotropy can be completely erased by another method which is a more complex than that described in this paper.

[17] Alloul, H., J. Appl. Phys. 50 (1979) 7330.

[18] MONOD, P. and COWEN, private communication.

[19] ARVANitis, D. and Fert, A., to be published. 